\title{
Relationship Between Rotavirus P[6] Infection in Korean Neonates and Histo-Blood Group Antigen: a Single-Center Study
}

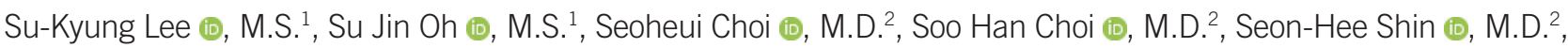

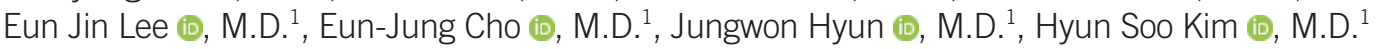 \\ ${ }^{1}$ Department of Laboratory Medicine, Hallym University Dongtan Sacred Heart Hospital, Hallym University College of Medicine, Hwaseong, Korea; \\ ${ }^{2}$ Department of Pediatrics, Hallym University Dongtan Sacred Heart Hospital, Hallym University College of Medicine, Hwaseong, Korea
}

Background: Rotaviruses are a major cause of pediatric gastroenteritis. The rotavirus P[6] genotype is the most prevalent genotype isolated from Korean neonates but has rarely been reported in other countries. Histo-blood group antigen (HBGA) is known to play an important role in rotavirus infection. We investigated the relationship between rotavirus genotype and HBGA-Lewis blood type in Korean children and explored the reasons for the predominance of rotavirus P[6] strain in Korean neonates.

Methods: Blood and stool samples were collected from 16 rotavirus-infected patients. Rotavirus G (VP7) and P (VP4) genotyping was performed using reverse transcription-PCR and sequencing. Lewis antigen phenotypes $\left(\mathrm{Le}^{\mathrm{a}} / \mathrm{Le}^{\mathrm{b}}\right)$ were tested, and HBGA-Lewis genotype was determined by sequencing the secretor (FUT2) and Lewis (FUT3) genes. Deduced amino acid sequences and three-dimensional structures of the VP8* portion of the rotavirus VP4 protein were analyzed.

Results: All P[6] rotaviruses were isolated from neonates under one month of age, who were negative or weakly positive for the Le ${ }^{b}$ antigen. However, 10 of the 11 non-P[6] rotaviruses were isolated from older children who were Le ${ }^{\mathrm{b}}$ antigen-positive. The VP8* amino acid sequences differed among P[6], P[4], and P[8] genotypes. Korean P[6] strains showed a unique VP8* sequence with amino acid substitutions, including Y169>L169, which differed from the sequences of P[6] strains from other countries.

Conclusions: The predominance of the rotavirus P[6] genotype in Korean neonates may be related to the interaction between HBGA-Lewis antigen and the VP8* portion of the VP4 protein, and this information will be helpful in future neonatal vaccine development.

Key Words: Rotavirus, Neonate, Genotype, Lewis, Histo-blood group antigen, Korean; VP4 protein
Received: February 18, 2020

Revision received: May 5, 2020

Accepted: September 24, 2020

\section{Corresponding author:}

Hyun Soo Kim, M.D., Ph.D.

Department of Laboratory Medicine, Hallym University Dongtan Sacred Heart Hospital, Hallym University College of Medicine, 7 Keunjaebong-gil, Hwaseong, Gyeonggido 18450 , Korea

Tel: +82-31-8086-2775

Fax: +82-31-8086-2789

E-mail: hskim0901@empas.com

\section{INTRODUCTION}

Rotaviruses are one of the major causes of pediatric gastroenteritis worldwide. Rotaviruses cause $5-10 \%$ of all cases of acute gastroenteritis in young children of $<5$ years of age, and $30 \%$ of rotavirus infections are severe enough to require treatment [1].
Rotavirus is a non-enveloped, double-stranded RNA (dsRNA) virus with a triple-layered capsid surrounding a genome of 11 dsRNA segments [1]. The outer capsid layer comprises two structural proteins, VP7 and VP4; based on these, rotaviruses are classified into at least $36 \mathrm{G}$ and $51 \mathrm{P}$ genotypes [2]. G1-G4 and $G 9$ and $P[4], P[6]$, and $P[8]$ are the most prevelent $G$ and $P$ 
genotypes, respectively [1, 3], and the genotypic distribution of rotavirus strains shows temporal and geographical fluctuations $[1,4]$.

In Korea, G1P[8] is the most prevalent genotype in children, whereas G4P[6] is the most prevalent genotype in neonates [3, 5-8]. The predominance of the G4P[6] and the G8P[6] genotype in Korean neonates has been reported from 1999 to 2019 [5-8]. Notably, the predominance of G4P[6] in neonates has not been reported in other countries. The rotavirus P[6] genotype is mainly isolated from Korean neonates, but is rarely isolated in older children [5-8].

Recently, rotavirus infection mediated by the attachment of the VP8* domain of the VP4 spike protein to specific histoblood group antigens (HBGAs) in the gut epithelium has been reported [9-11]. HBGAs are blood-type determinants determined by an individual's secretor gene (FUT2, fucosyltransferase 2) and Lewis gene (FUT3, fucosyltransferase 3) [12].

We investigated the relationship between rotavirus genotype and HBGA-Lewis type in Korean children and explored the reasons for the predominance of rotavirus P[6] infection in Korean neonates.

\section{MATERIALS AND METHODS}

\section{Patient samples}

We used rotavirus-positive stool samples and corresponding blood samples, which were remnants to be discarded after rotavirus test and complete blood count test, collected from acute gastroenteritis patients at Hallym University Dongtan Sacred Heart Hospital, Hwaseong-si, Korea, between February 2019 and January 2020. The rotavirus antigen test was conducted using SD BIOLINE Rotavirus (SD BIOLINE Rotavirus; Abbott Diagnostic Korea, Yongin, Korea) or RIDASCREEN Rotavirus test kits (RIDASCREEN Rotavirus; R-Biopharm, Darmstadt, Germany), according to the manufacturer's protocol. The Lewis phenotyping test and DNA extraction were performed on day of sample collection; extracted DNA and remnant diluted stool samples were stored at $-70^{\circ} \mathrm{C}$ until use. During this period, 66 of the 726 stool samples $(9.1 \%)$ were rotavirus-positive; adequate stool and corresponding blood samples for sequencing the four genes (the rotavirus VP4NP7 genes and FUT2/FUT3 genes) and Lewis antigen phenotyping were available for 16 patients. Median patient age was 3.4 years (range, 8 days -10 years), and six patients (37.5\%) were under 30 days of age. The Institutional Review Board (IRB) of Hallym University Dongtan Sacred Heart Hospital (IRB No. 2017-08-007) approved the study protocol and waived the need for informed consent.

\section{Rotavirus $\mathrm{G}$ and $\mathrm{P}$ genotyping}

Rotavirus G (VP7) and P (VP4) genotyping was performed using reverse transcription-PCR and sequencing. Viral RNA was extracted from diluted stool samples using the QIAamp Viral RNA Mini kit (Qiagen, Hilden, Germany) and the QIAcube platform (Qiagen). The RNA was denatured and reverse-transcribed using the SuperScript III First-Strand Synthesis System (Invitrogen, Carlsbad, CA, USA). The VP7 and VP4 genes were amplified from the dsRNA genome using specific primer sets, VP7-FNP7$\mathrm{R}$ and VP4-FNP4-R, respectively $[7,8,13]$. PCR products were visualized by electrophoresis on agarose gels and analyzed by DNA sequencing using ABI BigDye Terminator v3.1 Cycle Sequencing kits (Applied Biosystems, Foster City, CA, USA) and an ABI 3500 XL DNA Analyzer (Applied Biosystems). Genotypes were confirmed using the Basic Local Alignment Search Tool on the National Center for Biotechnology Information (https://blast.ncbi.nlm.nih.gov/)

\section{Lewis antigen phenotyping}

Lewis antigen phenotyping was performed using ID-Antigen Profile I (Bio-Rad, Cressier Switzerland) and gel suspensions containing antibodies for the $\mathrm{P}, \mathrm{Le}^{\mathrm{a}}, \mathrm{Le}^{\mathrm{b}}, \mathrm{Lu}^{\mathrm{a}}, \mathrm{Lu}^{\mathrm{b}}$, and ctl antigens, according to the manufacturer's protocol. A 5\% erythrocyte suspension was prepared from each EDTA-anticoagulated blood sample by mixing $50 \mu \mathrm{L}$ of erythrocytes in $0.5 \mathrm{~mL}$ of Diluent 1 solution. The cell suspension was incubated for 10 minutes at room temperature $\left(18-25^{\circ} \mathrm{C}\right)$, and $12.5 \mu \mathrm{L}$ of the cell suspension was added to each phenotype ID-card micro-tube. The ID-cards were then centrifuged for 10 minutes in the IDcentrifuge $12 \mathrm{~S} \mathrm{II} \mathrm{(Bio-Rad)} \mathrm{and} \mathrm{read} \mathrm{in} \mathrm{the} \mathrm{range} \mathrm{between} \mathrm{neg-}$ ative to $4+$, according to the agglutination strength.

\section{PCR and sequencing of the secretor and Lewis genes (FUT2 and FUT3)}

DNA was extracted from EDTA-anticoagulated blood samples using the QIAamp Blood Mini kit (Qiagen). A 1,153-bp region of the FUT2 gene was amplified using the tk1/tk2 primer set [14], and two segments (917 and $419 \mathrm{bp}$ ) of the FUT3 gene were amplified using two primer sets (N-F1/6R and 4F/8R) [15]. The forward primer sequence of N-F1 (TGATGACCGGCTATGCCTTC) was specifically designed for this study. PCR products were analyzed by DNA sequencing using ABI BigDye Terminator v3.1 Cycle Sequencing kits and an ABI 3500 XL DNA Analyzer. Variants of the FUT2 and FUT3 genes were detected by compari- 
son with reference sequences FUT2*01 and FUT3 (http://erythrogene.com). Minor allele variants at the FUT2 c.418, c.461, and c.772 and FUT3 c.59, c.202, c.314, c.508, and c.1067 sites, which are known to be related to Lewis antigen phenotypes, were examined [16, 17].

Table 1. Rotavirus genotype, erythrocyte Lewis antigen phenotype, and FUT2/FUT3 gene variants in patients with rotavirus infection

\begin{tabular}{|c|c|c|c|c|c|}
\hline $\begin{array}{l}\text { Rotavirus } \\
\text { genotype }\end{array}$ & $\begin{array}{c}\text { Case } \\
\text { number }\end{array}$ & Sex/Age & $\begin{array}{c}\text { Erythrocyte } \\
\text { Lewis } \\
\text { phenotype }\end{array}$ & $\begin{array}{c}\text { Minor allele } \\
\text { homozygosity } \\
\text { (FUT2 c. } 418 \text {, } \\
\text { c.461, c. } 772 \text { ) }\end{array}$ & $\begin{array}{c}\text { Minor allele } \\
\text { homozygosity } \\
\text { (FUT3 c.59, } \\
\text { c. } 202 \text {, c. } 314 \text {, } \\
\text { c. } 508 \text {, or } \\
\text { c. } 1067 \text { ) }\end{array}$ \\
\hline \multirow[t]{5}{*}{ G8P $[6]$} & $\begin{array}{c}1 \\
\text { 19RP-09 }\end{array}$ & M/15 days & $\operatorname{Le}^{\mathrm{a}}(3+) \operatorname{Le} \mathrm{e}^{\mathrm{b}}(-)^{*}$ & c. $418 \mathrm{~A}>\mathrm{T}$ & - \\
\hline & $\begin{array}{c}2 \\
\text { 19RP-11 }\end{array}$ & M/8 days & $\operatorname{Le}^{\mathrm{a}}(1+) \operatorname{Le}(2+)$ & - & - \\
\hline & $\begin{array}{c}3 \\
\text { 19RP-26 }\end{array}$ & M/20 days & $\operatorname{Le}^{\mathrm{a}}(-) \operatorname{Le}^{\mathrm{b}}(-)$ & - & c. $508 \mathrm{G}>\mathrm{A}$ \\
\hline & $\begin{array}{c}4 \\
\text { 19RP-35 }\end{array}$ & M/15 days & $\operatorname{Le}^{\mathrm{a}}(-) \mathrm{Le}^{\mathrm{b}}(-)$ & c. $418 \mathrm{~A}>\mathrm{T}$ & c.59T $>\mathrm{G}$ \\
\hline & $\begin{array}{c}5 \\
\text { 19RP-38 }\end{array}$ & M/9 days & $\operatorname{Le}^{\mathrm{a}}(-) \operatorname{Le}(-)$ & - & $\begin{array}{l}\text { c. } 59 \mathrm{~T}>\mathrm{G} \\
\text { c. } 508 \mathrm{G}>\mathrm{A}\end{array}$ \\
\hline G1P[8] & $\begin{array}{c}6 \\
\text { 19RP-23 }\end{array}$ & $\mathrm{F} / 3 \mathrm{yr}$ & $L e^{a}(-) L e^{b}(4+)$ & - & - \\
\hline \multirow[t]{3}{*}{ G8P[8] } & $\begin{array}{c}7 \\
\text { 19RP-04 }\end{array}$ & $\mathrm{M} / 6 \mathrm{yr}$ & $L e^{a}(-) L e^{b}(4+)$ & - & - \\
\hline & $\begin{array}{c}8 \\
\text { 19RP-07 }\end{array}$ & $\mathrm{M} / 4 \mathrm{yr}$ & $\operatorname{Le}^{\mathrm{a}}(-) \operatorname{Le}(4+)$ & - & - \\
\hline & $\begin{array}{c}9 \\
\text { 19RP-31 }\end{array}$ & $\mathrm{F} / 4 \mathrm{yr}$ & $\operatorname{Le}^{\mathrm{a}}(-) \operatorname{Le}(4+)$ & - & - \\
\hline \multirow[t]{3}{*}{ G9P[8] } & $\begin{array}{c}10 \\
\text { 19RP-02 }\end{array}$ & $\mathrm{F} / 3 \mathrm{yr}$ & $\operatorname{Le}^{\mathrm{a}}(-) \mathrm{Le}^{\mathrm{b}}(4+)$ & - & - \\
\hline & $\begin{array}{c}11 \\
\text { 19RP-15 }\end{array}$ & $\mathrm{F} / 6 \mathrm{yr}$ & $\operatorname{Le}^{a}(-) \operatorname{Le}^{b}(3+)$ & - & - \\
\hline & $\begin{array}{c}12 \\
\text { 19RP-24 }\end{array}$ & F/ 20 days & $\operatorname{Le}^{a}(3+) \operatorname{Le}^{b}(3+)$ & - & - \\
\hline \multirow[t]{4}{*}{$\mathrm{G} 2 \mathrm{P}[4]$} & $\begin{array}{c}13 \\
\text { 19RP-13 }\end{array}$ & $\mathrm{M} / 5 \mathrm{yr}$ & $\operatorname{Le}^{\mathrm{a}}(-) \operatorname{Le}^{\mathrm{b}}(4+)$ & - & - \\
\hline & $\begin{array}{c}14 \\
\text { 19RP-17 }\end{array}$ & $\mathrm{F} / 10 \mathrm{yr}$ & $\operatorname{Le}^{\mathrm{a}}(-) \mathrm{Le}^{\mathrm{b}}(3+)$ & - & - \\
\hline & $\begin{array}{c}15 \\
\text { 19RP-21 }\end{array}$ & F/1 yr & $\operatorname{Le}^{\mathrm{a}}(-) \operatorname{Le}^{\mathrm{b}}(4+)$ & - & - \\
\hline & $\begin{array}{c}16 \\
19 R P-32\end{array}$ & $\mathrm{M} / 9 \mathrm{yr}$ & $\operatorname{Le}^{a}(-) \operatorname{Le}(3+)$ & - & - \\
\hline
\end{tabular}

Abbreviations: $M$, male; F, female.

*Agglutination strength was expressed in the range of - to 4+, according to the manufacturer's guidelines.
Analyses of amino acid sequences and three-dimensional (3D) structure of VP8*

The deduced amino acid sequences of the rotavirus VP8* portion of the VP4 capsid protein were analyzed and compared with the reference VP4 sequence data from GenBank: Wa_G1P[8]_ JX406750, DS-1_G2P[4]_HQ650119, RV3_P[6]_U16299, and $\mathrm{P}[6]$ strains detected in other countries (JN129097, LC095922, KF835915, and KF726067) and in Korea (MK953605 and G8P[6] from a previous study [8]).

Multiple sequence alignment and translation to amino acids were conducted using the MEGA version 7 program [18]. The 3D structures of the deduced amino acid sequences of the VP8* portion were obtained using the Swiss-Model program [19], PDB (Protein Data Bank. http://www.rcsb.org/) [20], and UCSF Chimera Program [21]. Briefly, the deduced amino acid sequences of the VP8* portion were converted to PDB format files in the Swiss-Model program, and structure comparison analyses were performed using the UCSF Chimera Program using the converted PDB files.

\section{Statistical analysis}

The relationship between $\mathrm{P}[6]$ and $\mathrm{Le}^{\mathrm{b}}$ antigen negativity is expressed as a $2 \times 2$ contingency table, and $P$ was calculated by Fisher's exact test using MedCalc software version 19 (MedCalc Sofware Ltd, Ostend, Belgium). $P<0.05$ was considered statistically significant.

\section{RESULTS}

Rotavirus genotype, erythrocyte Lewis antigen phenotypes, and FUT2/FUT3 gene variants in rotavirus infected patients

Table 1 shows the rotavirus genotyping, Lewis antigen phenotyping, and FUT2/FUT3 gene results. Of the 16 rotavirus-positive patients, five patients under one month of age were positive for P[6] (all G8P[6]) rotaviruses; these patients were negative (patients $1,3,4$, and 5) or weakly positive (patient 2) for the Le ${ }^{b}$ antigen. In contrast, non-P[6] rotaviruses (G2P[4]s, G8P[8]s, G9P[8]s, and G1P[8]) were isolated from older children (except

Table 2. Relationship between rotavirus P[6] genotype and Le $e^{b}$ antigen negativity in patients with rotavirus infection

\begin{tabular}{llcc}
\hline Rotavirus genotype & $\mathrm{Le}^{\mathrm{b}}(-)$ & $\mathrm{Le}^{\mathrm{b}}(+)$ & $P$ \\
\hline $\mathrm{P}[6]^{*}(\mathrm{~N}=5)$ & $4(80 \%)$ & $1(20 \%)$ & 0.0027 \\
Non-P[6] $]^{*}(\mathrm{~N}=11)$ & $0(0 \%)$ & $11(100 \%)$ &
\end{tabular}

${ }^{*}$ All P[6] rotaviruses were isolated from neonates under one month of age, and 10 of the 11 non-P[6] rotaviruses were isolated from older children. 


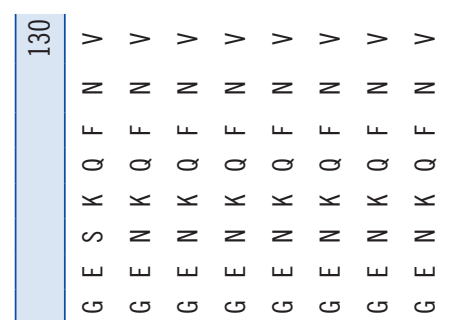

I $->>>>>$

$-z z z z z z z$

$>>>>>>$

○ 0 o 0 o 0000

$\simeq \propto \propto \propto \propto \propto \propto \propto ⿻ 上 丨$

00000000

$>>>>>>>$

a a a a a a a a

20000000

$\exists \pm \pm x \pm x \pm \pm x$

a a a a a a a a

ம

$->>>>>$

$<<<<<<<<$

$>->-1-1-$
$>>>>>>$

$<<<<<<<<$

33333333

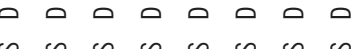

$z z z z z z z$

$z z z z z z z$

$\vdash \vdash \vdash \vdash \vdash \vdash \vdash$

es en en en en es es es

س

$>>>>>>$

$>>>>>>>$
ब $>>>>>$

ज 0000000

$z z z z z z z$

$\vdash \vdash \vdash \vdash \vdash \vdash$

$z z z z z z z$

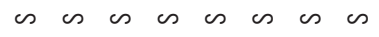

$z z z z z z z$

$-------$

\lrcorner$-\sqcup-\sqcup--\sqcup$

क $33 \leq 3 \leq 3 \leq 3$

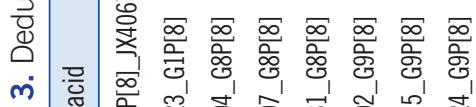

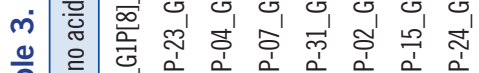

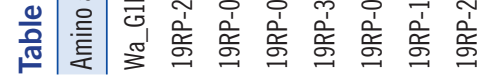

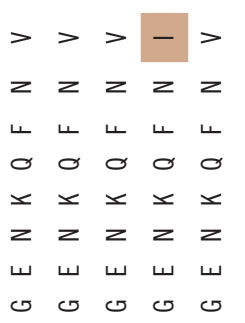

ப ா ய ய

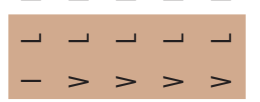

$>>>>$

○ 0000

$\propto \propto \propto \propto \propto ⿻ 上 丨$

$z z z z z$

$\vdash \vdash \vdash \vdash$

o a a o a

$\sim \sim \sim \sim$
$>>>$

I $I I x$

$\circ \circ \circ \circ \circ$

س

$>>>>$

$\varangle<\ll<$

- - - -

$>>>>$

$\varangle<\ll<\ll$

$\vdash \vdash \vdash \vdash$

3333

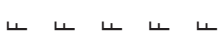

○ 0000

$z$ as as us

$z z z z z$

$z z z z$

$\vdash \vdash \vdash \vdash$

es es es es

س

$>>>>$

$>>>>$

$>>>>$

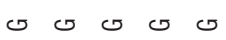

$z 0000$

$\vdash \vdash \vdash \vdash$

$z z z z z$

as as en en

an en en en

$----$

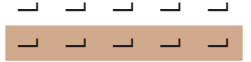

$3 z 33$

蓉

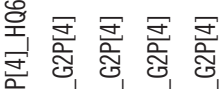

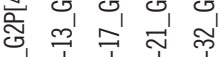

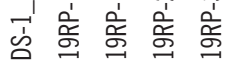
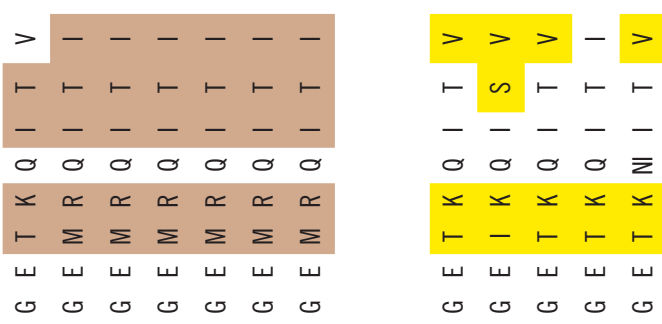

பா ப ப ப

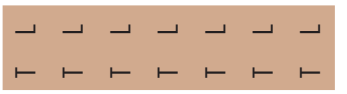

$>>>>>>$

○ 000000

$\propto \propto \propto \propto \propto \propto ⿻ \propto ⿻ 上 丨$

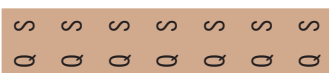

$z z z z z z$

$\vdash \vdash \vdash \vdash \vdash$

$>>>>>>$

$z z z z z z$

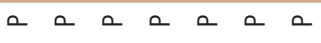

山

$>>>>>>$

$\begin{array}{lllllll}\bullet & - & - & - & - & - & - \\ \bullet & - & - & - & - & - & -\end{array}$

$\neg-\sqcup-\neg-\sqcup$

$<<<<<<<$

333333

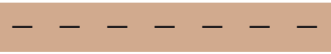

0000000

$\vdash \vdash \vdash \vdash \vdash \vdash$

$x x x x x x$

$z z z z z z z$

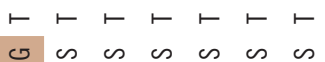

س

$\neg-\neg-\neg-\sqcup$

$>>>>>>$

$>>>>>>$

0000000

○ 000000

$z 000000$

$\vdash \vdash \vdash \vdash \vdash \vdash$

$\therefore \circ a \quad a \quad a \quad a \quad a$

$z z z z z z$

$-\downarrow-\downarrow-\downarrow-$

$\neg-\sqcup-\neg-\sqcup$

ப ா ய

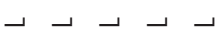

$\vdash \vdash \vdash \vdash$

$>>>>$

○ 000

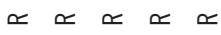

as en un en

○ 0000

$z$ s $z z z$

ค $\vdash ー \vdash$

$>\vdash>->$

$z z z z$

$\circ \circ \circ \circ \circ$

س

$>--->$

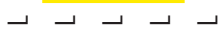

$\rightarrow-\longrightarrow--$

$-\rightarrow---$

$\ll<\ll<\ll$

$>>>>$

3333

$->>>$

- 0 o 0 .

$\vdash \vdash \vdash \ll$

$\propto \propto \propto \propto \propto ⿻ 上 丨$

$z z z z z$

$\vdash \vdash \vdash \vdash$

उण丁ण

山

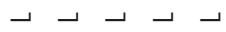

$>>>>$

$>>>>$

$0<0 x \sigma$

○ 000

$z z z z z$

$\vdash \vdash \vdash \vdash$

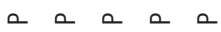

$z z \infty z \infty$

$-\rightarrow-\rightarrow-$

$\rightarrow-\rightarrow-\rightarrow$

33333

$----1$

3333

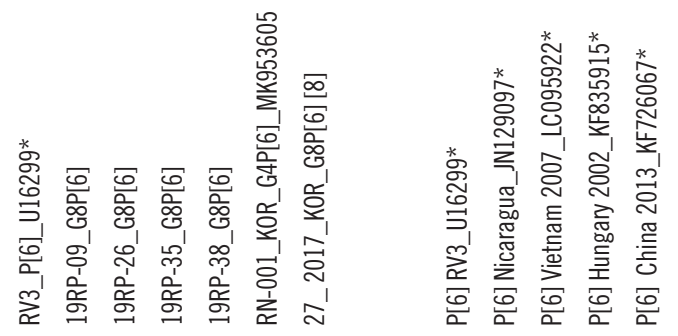


总

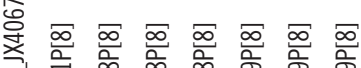

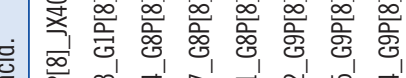

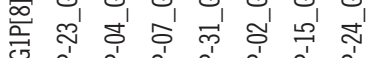

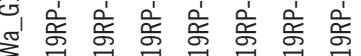

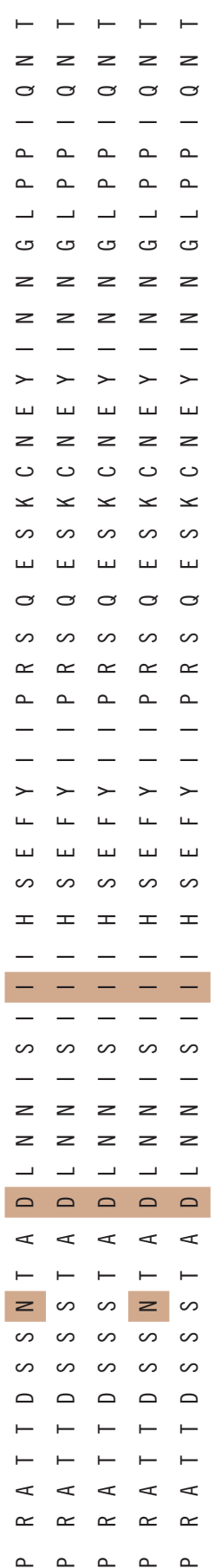

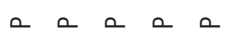

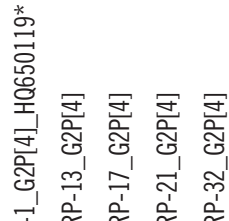

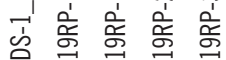

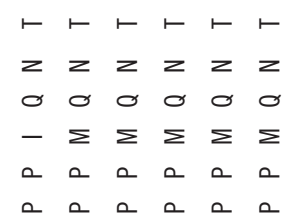

$\circ \circ a \quad a \quad 0 \quad 0$

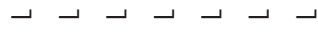

丁ण丁丁ण丁丁

$1 z z z z-z$

$z z z z z z$

$------$

$>>>>>$

ш

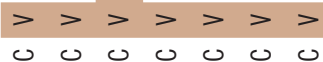
$x x x \leq x \leq x$

us es es es es es es

山

○ 0 ○ 0 ○ 00

us en es as us en es

$\propto \propto \propto \propto ⿻ \propto ⿻$

a a a a a a a

$------$

$------$

$>>>>>$

ப ப ப ப ப

山

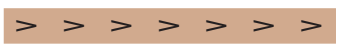

I $\mp-\rightarrow$ I

$\neg-\downarrow-\downarrow$

$\circlearrowleft \circlearrowleft \circlearrowleft \circlearrowleft \circlearrowleft$

$\vdash \vdash \vdash \vdash$

$z z z z$

$----$

$>>>>$

山

$>z z x z$

00000

$x x x \leq x$

es es en es en

ए

00000

us us us us

$\propto \propto \propto \propto ⿻ 上$

¿ a a a a

$----$

$--->$
$>>>>$

ப ப L L L

س س ا

$>\ll \vdash \vdash \vdash$

I I I

$-\quad-\quad-\quad-\quad-$
$\vdash>>>>$

$1<<<<<<$

ம

$>>>>>>$

山

es es es en as en es

$\neg \sqcup-\neg \sqcup-\sqcup$

$z z z z z z$

as as en en as en en

$\vdash \vdash \vdash \vdash \vdash \vdash$

$\infty \sqcup-\neg-\sqcup-$

$\infty\lrcorner-\downarrow-\downarrow-$

$>>>>>$

0000000

$\vdash \vdash \vdash \vdash \vdash \vdash$

$\vdash \vdash \vdash \vdash \vdash \vdash$

$<<<<<<<$

I I I I I I

a a a a a a a

-----
$\vdash>>>$

$\vdash \vdash \vdash \vdash$

ш

$>-->>$

$\omega>>0>$

凹 $山$ ए

- en en en en

$z-\downarrow-\downarrow$

s $z z z z$

- en en es es

$\omega \vdash \omega \vdash \omega$

us es us as

$>>>>$

00000

$\vdash \vdash \vdash \vdash$

$\vdash \vdash \vdash \vdash$

$<<<<<$

$x \pm x z \pm$

$\circ$ a a a a

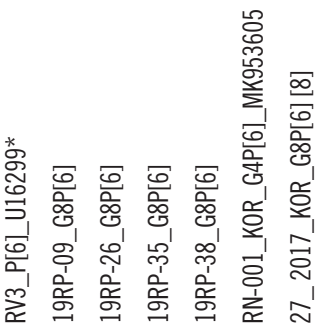


patient 12 , aged 20 days); these patients were Le $e^{\mathrm{b}}$ antigen-positive. Table 2 shows the relationship between P[6] rotavirus and $\mathrm{Le}^{\mathrm{b}}$ antigen negativity. The patients with $\mathrm{Le}^{\mathrm{b}}$ antigen-negative phenotypes (patients 1, 3, 4, and 5) showed FUT2 c.418A > T homozygosity, FUT3 c.59T > G homozygosity, or FUT3 c.508G> A homozygosity. The patients with $\mathrm{Le}^{\mathrm{b}}$ antigen-positive phenotypes did not show FUT2 c.418A>T homozygosity, FUT3 c.59T>G homozygosity, or FUT3 c.508G > A homozygosity. Several other variants were also detected in the FUT2 and FUT3 genes; however, we focused on the FUT2 c.418, c.461, and c.772 and FUT3 c.59, c.202, c.314, c.508, and c.1067 sites.

\section{Amino acid sequence of the VP8* portion of the VP4 capsid protein}

The amino acid sequences of the VP8* portion of the P[8], P[4], and $P[6]$ genotypes greatly varied according to the genotype (Table 3). The amino acid sequences of the VP8* portion of Korean P[6] strains (19RP-09_G8P[6], 19RP-26_G8P[6], 19RP35 G8P[6], 19RP-38 G8P[6], RN-001_KOR_G4P[6], and 27_2017_KOR_G8P[6]) were notably different from those of $P[6]$ strains detected in other countries (P[6] RV3, P [6] Nicaragua, P[6] Vietnam, P[6] Hungary, and P[6] China). The amino acid differences between P[6] strains from other countries and Korean P[6] strains were as follows: N88D, V103I, T125M, K126R, K136T, S145R, S146T, V147A, S148G, R155H, H169L, H178Y, and S188L S189L T198A (yellow shading in Table 3). We omitted the 19RP-11 data in Table 3, because this portion (amino acids 81-230 in Table 3) of the VP4 sequence of 19RP-11 yielded an unclear sequence chromatogram.

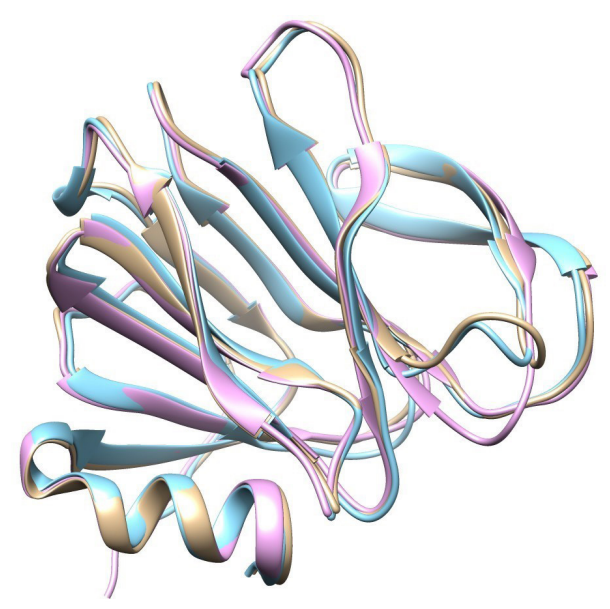

Fig. 1. Superposition of P[6] VP8*, P[4] VP8*, and P[8] VP8* structures deduced from the VP4 nucleotide sequences. Sky blue, $\mathrm{P}[6]$; gold, $\mathrm{P}[8]$; and pink, $\mathrm{P}[4]$.

\section{D structure of the VP8* glycoprotein}

Fig. 1 shows the superposition of the P[6] VP8*, P[4] VP8*, and P[8] VP8* structures deduced from the VP4 amino acid sequences of $P[6], P[4]$, and $P[8]$ strains. Rotaviruses with similar genotypes showed nearly the same 3D structures of the VP8* portion, although one or two amino-acid sequence differences were observed. Despite the differences in amino acid sequences at various sites between P[4], P[6], and P[8], only a slight structural difference was observed.

\section{DISCUSSION}

We investigated the relationship between rotavirus genotypes and HBGA-Lewis blood type in Korean children with rotavirus infections, and explored the reason for the P[6] genotype predominance in Korean neonates. Although the number of cases was small, all P[6] rotaviruses were isolated from neonates who were $\mathrm{Le}^{\mathrm{b}}$ antigen-negative or weakly positive. In contrast, nonP[6] rotaviruses were isolated from older children (except for patient 12, aged 20 days) who were Le ${ }^{b}$ antigen-positive. These results suggest that neonate-specific P[6] rotaviruses easily infect neonates who are negative or weakly positive for $\mathrm{Le}^{\mathrm{b}}$ antigen, but rarely infect patients who are $\mathrm{Le}^{\mathrm{b}}$ antigen-positive or older children. Our results are supported by recent studies showing that rotavirus P[6] strains can infect Lewis-negative individuals, regardless of their secretor status [22, 23]. In the neonate gut, modification to the precursor moieties or branching of Lewis HBGA is developmentally regulated, and thus the most abundant type of Lewis HBGA in the neonate gut is the unbranched type I precursor glycan, Le(a-b-) [24]. Only a few studies in Africa have also reported the relationship between $\mathrm{P}[6]$ and neonates; however, the proportion of $\mathrm{P}[6]$ infection in neonates were at most $25 \%$ in those studies [22, 25, 26]. This phenomenon may be related to the higher proportion of Le(ab-) in Koreans and Africans than in Caucasian or Chinese populations [14, 22, 27, 28]. Contrarily, this may be related to the noted differences in the VP8* amino acid sequences between the Korean rotavirus P[6] strains and those of the P[6] strains detected in other countries (Table 3).

In terms of differences in the 3D structure between the rotavirus VP8* portion and Lewis HBGA, the cleft between the betasheets of rotavirus $P[6]$ has been reported to be wider than that in P[14], and rotavirus P[6] has been suggested to bind to the unbranched type I precursor glycan, Le(a-b-), rather than to the branched HBGA, Le(a-b+) [12]. However, in our study, the cleft between the beta-sheets of the P[6] rotavirus was not markedly 
wider than that of $\mathrm{P}[4]$ or $\mathrm{P}[8]$ strains (Fig. 1).

A previous study suggested that a single residue of the neonatal P[6] VP8*, specifically H169, which is well conserved in most neonate-specific P[6] genotypes, alters the glycan conformation [12]. However, all neonatal G8P[6] in this study showed a different amino acid residue, L169 instead of H169, which can cause P[6] infection in Korean neonates. Other amino acid substitutions at different sites (N88D, V103I, T125M, K126R, K136T, S145R, S146T, V147A, S148G, R155H, H169L, H178Y, S188L S189L, and T198A) may cause a small change in the 3D structure or hydrogen bond/hydrophobic interaction between the VP8* of the rotavirus VP4 protein and HBGA molecules in patients, which may involve age- or population-related tropism of some P[6] strains [12].

The Lewis histo-blood group system consists of two major antigens, Le $e^{a}$ and Le $e^{b}$, and four phenotypes, Le(a-b-), Le(a+b-), Le $(a-b+)$, and Le $(a+b+)$ [29]. Lewis antigen synthesis depends on the interaction of FUT3 and FUT2. Although the number of cases in our study was too small to reveal the relationship between phenotype and genotype, our results are compatible with previous interpretations of Lewis genotypes $[16,17]$. In terms of FUT2, patients harboring homozygous G428A (c.461) and G739A (c.772) variants were non-secretors, and those with a homozygous A385T (c.418) variants were weak secretors. Patients who were wild-type or harbored heterozygous variants at these sites were designated as secretors. In terms of FUT3, patients who harbored homozygous variants for at least T202C (c.202), G508A (c.508), or T1067A (c.1067) were designated as Le(a-b-) [16]. In another study, patients with wild-type FUT2

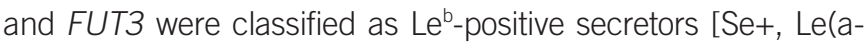
$\mathrm{b}+)$ ]; patients who were homozygous for the minor allele in FUT2 at rs601338 (G428A, c.461) and/or rs602662 (G739A, c.772), with a wild-type FUT3 genotype were classified as Le ${ }^{\mathrm{b}}$-negative non-secretors [Se-, Le(a+b-)]; patients homozygous for the minor allele in FUT3 at rs778986 (C314T, c.314), rs28362459 (T59G, c.59), rs3894326 (T1067A, c.1067), and/or rs3745635 (G508A, c.508), with a wild-type FUT2 genotype were considered to have a secretor Lewis-null phenotype [Se+, Le(a-b-)]; and those homozygous for the minor allele at one or more sites in FUT2 and FUT3 were considered to have a non-secretor Lewis-null phenotype [Se-, Le(a-b-)] [17]. To the best of our knowledge, the relationship between all Lewis gene variants and the Lewis antigen phenotypes has not been clearly established and thus, there were slight differences in interpretation across the studies. Therefore, we focused on the FUT2 c.418, c.461, and c.772 and the FUT3 c.59, c.202, c.314, c.508, and c.1067

sites. Further studies will be needed to elucidate the relationship between all variants of Lewis-related genes and Lewis antigen phenotypes.

In conclusion, we found that the rotavirus P[6] genotype predominance in Korean neonates may be related to the interaction between the patient's HBGA-Lewis antigen phenotype and the VP8* portion of the rotavirus VP4 capsid protein. Four possible explanations are suggested: (1) P[6] infection may be related to Le $^{b}$ antigen-negative or weakly positive status; (2) Lewis antigens are not fully developed in the neonate gut; (3) the proportion of Le(a-b-) is higher in Korea than in other countries; and (4) the antigenicity of rotavirus P[6] strains isolated in Korea is different from that of P[6] strains isolated in other countries. Although, this study is limited with the small number of patients, the results provide valuable information on future development of neonatal rotavirus vaccine.

\section{ACKNOWLEDGEMENTS}

We thank Jeong Hee Kim for technical assistance.

\section{AUTHOR CONTRIBUTIONS}

HSK designed and performed the study and wrote the manuscript; SKL performed the experiment and helped to wrote the manuscript; SJO collected and analyzed the data; SHC and SHS obtained the patients' samples and clinical information; EJL, EJC, and JH helped to analyze the data; All authors read and approved the final manuscript.

\section{CONFLICTS OF INTEREST}

No potential conflicts of interest relevant to this study are reported.

\section{RESEARCH FUNDING}

This work was supported by the National Research Foundation of Korea (NRF-2017R1D1A3B03031940) and by the Hallym University Research Fund.

\section{ORCID}

Su-Kyung Lee

Su Jin Oh

Seoheui Choi
https://orcid.org/0000-0002-2536-413X https://orcid.org/0000-0002-8650-7296 https://orcid.org/0000-0001-7515-0554 
Soo Han Choi

Seon-Hee Shin

Eun Jin Lee

Eun-Jung Cho

Jungwon Hyun

Hyun Soo Kim

\section{REFERENCES}

1. Desselberger U. Rotaviruses. Virus Res 2014;190:75-96.

2. Rotavirus Classification Working Group: RCWG-Laboratory of Viral Metagenomics. https://rega.kuleuven.be/cev/viralmetagenomics/virusclassification/rcwg (Updated on 16 Feb 2020).

3. Than VT and Kim W. Prevalence of rotavirus genotypes in South Korea in 1989-2009: implications for a nationwide rotavirus vaccine program. Korean J Pediatr 2013;56:465-73.

4. Kawai K, O'Brien MA, Goveia MG, Mast TC, El Khoury AC. Burden of rotavirus gastroenteritis and distribution of rotavirus strains in Asia: a systematic review. Vaccine 2012;30:1244-54.

5. Kim JS, Kim HS, Hyun J, Kim HS, Song W, Lee KM, et al. Analysis of rotavirus genotypes in Korea during 2013: an increase in the G2P[4] genotype after the introduction of rotavirus vaccines. Vaccine 2014;32: 6396-402.

6. Kim JS, Kim SM, Kim HS. High prevalence of rotavirus G4P[6] genotypes among neonates in two Korean hospitals. Ann Clin Microbiol 2017;20:63-6.

7. Lee SK, Choi S, Kim JS, Lee EJ, Hyun J, Kim HS. Whole-genome analysis of rotavirus G4P[6] strains isolated from Korean neonates: association of Korean neonates and rotavirus P[6] genotypes. Gut Pathog 2019;11:37.

8. Lee SK, Choi S, Shin SH, Lee EJ, Hyun J, Kim JS, et al. Emergence of G8P[6] rotavirus strains in Korean neonates. Gut Pathog 2018;10:27.

9. Gozalbo-Rovira R, Ciges-Tomas JR, Vila-Vicent S, Buesa J, Santiso-Bellón $\mathrm{C}$, Monedero $\mathrm{V}$, et al. Unraveling the role of the secretor antigen in human rotavirus attachment to histo-blood group antigens. PLoS Pathog 2019;15:e1007865.

10. Hu L, Crawford SE, Czako R, Cortes-Penfield NW, Smith DF, Le Pendu J, et al. Cell attachment protein VP8* of a human rotavirus specifically interacts with A-type histo-blood group antigen. Nature 2012;485:256-9.

11. Hu L, Sankaran B, Laucirica DR, Patil K, Salmen W, Ferreon ACM, et al. Glycan recognition in globally dominant human rotaviruses. Nat Commun 2018;9:2631.

12. Hong YJ, Hwang SM, Kim TS, Song EY, Park KU, Song J, et al. Significance of Lewis phenotyping using saliva and gastric tissue: comparison with the Lewis phenotype inferred from Lewis and secretor genotypes. Biomed Res Int 2014;2014:573652.

13. Kim JS, Lee SK, Ko DH, Hyun J, Kim HS, Kim JS, et al. Performance evaluation of the automated fluorescent immunoassay system rotavirus

assay in clinical samples. Ann Lab Med 2019;39:50-7.

14. Song SY, An SS, Ryu SW, Kim JS, Suh IB. Evaluation of the genotypes of the Lewis Blood Group in a Korean population using direct sequencing. Korean J Hematol 2008;43:34-42.

15. Serpa J, Almeida R, Oliveira C, Silva FS, Silva E, Reis C, et al. Lewis enzyme (alpha1-3/4 fucosyltransferase) polymorphisms do not explain the Lewis phenotype in the gastric mucosa of a Portuguese population. J Hum Genet 2003;48:183-9.

16. Boniface K, Byars SG, Cowley D, Kirkwood CD, Bines JE. Human neonatal rotavirus vaccine (RV3-BB) produces vaccine take irrespective of histo-blood group antigen status. J Infect Dis 2020;221:1070-8.

17. King JR, Varadé J, Hammarström L. Fucosyltransferase gene polymorphisms and Lewisb-negative status are frequent in Swedish newborns, with implications for infectious disease susceptibility and personalized medicine. J Pediatric Infect Dis Soc 2019;8:507-18.

18. Kumar S, Stecher G, Tamura K. MEGA7: molecular evolutionary genetics analysis version 7.0 for bigger datasets. Mol Biol Evol 2016;33: 1870-4.

19. Waterhouse A, Bertoni M, Bienert S, Studer G, Tauriello G, Gumienny R, et al. SWISS-MODEL: homology modelling of protein structures and complexes. Nucleic Acids Res 2018;46:W296-303.

20. Burley SK, Berman HM, Bhikadiya C, Bi C, Chen L, Di Costanzo L, et al. RCSB Protein Data Bank: biological macromolecular structures enabling research and education in fundamental biology, biomedicine, biotechnology and energy. Nucleic Acids Res 2019;47:D464-74.

21. Pettersen EF, Goddard TD, Huang CC, Couch GS, Greenblatt DM, Meng EC, et al. UCSF Chimera-a visualization system for exploratory research and analysis. J Comput Chem 2004;25:1605-12.

22. Nordgren J, Sharma S, Bucardo F, Nasir W, Günaydın G, Ouermi D, et al. Both Lewis and secretor status mediate susceptibility to rotavirus infections in a rotavirus genotype-dependent manner. Clin Infect Dis 2014;59:1567-73

23. Van Trang N, Vu HT, Le NT, Huang P, Jiang X, Anh DD. Association between norovirus and rotavirus infection and histo-blood group antigen types in Vietnamese children. J Clin Microbiol 2014;52:1366-74.

24. Ameno S, Kimura H, Ameno K, Zhang X, Kinoshita H, Kubota T, et al. Lewis and Secretor gene effects on Lewis antigen and postnatal development of Lewis blood type. Biol Neonate 2001;79:91-6.

25. Todd S, Page NA, Duncan Steele A, Peenze I, Cunliffe NA. Rotavirus strain types circulating in Africa: review of studies published during 1997-2006. J Infect Dis 2010;202(S1):S34-42.

26. Pager CT, Alexander JJ, Steele AD. South African G4P[6] asymptomatic and symptomatic neonatal rotavirus strains differ in their NSP4, VP8*, and VP7 genes. J Med Virol 2000;62:208-16.

27. Heylen E, Zeller M, Ciarlet M, Lawrence J, Steele D, Van Ranst M, et al. Human P[6] Rotaviruses from Sub-Saharan Africa and Southeast Asia are closely related to those of human P[4] and P[8] Rotaviruses circulating worldwide. J Infect Dis 2016;214:1039-49.

28. Park HD, Park KU, Song J, Ki CS, Han KS, Kim JQ. The relationship between Lewis/Secretor genotypes and serum carbohydrate antigen 19-9 levels in a Korean population. Korean J Lab Med 2010;30:51-7. 